\title{
Effect of Calcium on the Vasoactive Response of Arterioles to Light during Fluorescent Intravital Microscopy of the Tibialis Anterior Muscle of the Hamster
}

\author{
Louis M. Messina and Ane Gardner \\ Jobst Vascular Research Laboratories, Department of Surgery, Section of Vascular Surgery, University \\ of Michigan Medical Center, Ann Arbor, Michigan 48109
}

Received August 7, 1991

\begin{abstract}
Arterioles may undergo a transient vasoactive response when exposed to light during fluorescent intravital microscopy. We hypothesized that the type and frequency of the vasoactive responses by arterioles to light is calcium dependent. In order to test this hypothesis we quantitated the type and frequency of vasoactive responses by arterioles to light in the presence and absence of calcium within the suffusate bathing the muscle. In addition, we determined whether the presence or absence of calcium also influenced the reactivity of these arterioles to adenosine and norepinephrine. In separate experiments, we determined the effect of increases in suffusate calcium concentration on the resting diameter of arterioles. The concentration of calcium in the suffusate significantly influenced the frequency of lightinduced vasoactivity of the arterioles of the tibialis anterior muscle: a normal suffusate calcium concentration of $2 \times 10^{-3} M$ was associated with an incidence of light-induced vasoreactivity of $77 \%$ but decreased significantly to $58 \%$ when calcium was removed from the suffusate. Although the frequency of the vasoactive response to light was different for the two experimental conditions, the type of vasoactive response, predominately vasomotion, was similar. The vasoactive responses of arterioles to adenosine and norepinephrine were similar in the presence and absence of calcium in the suffusate. However, the concentration of calcium in the suffusate did significantly influence resting arteriolar diameters: $2 \times 10^{-3}$ $M$ calcium $\mathrm{CaCl}_{2}$ caused a mean decrease in the arteriolar diameter of $11.6( \pm 3.0) \%, 4 \times$ $10^{-3} \mathrm{M} \mathrm{CaCl}_{2}$ caused a mean decrease of $41.2( \pm 12) \%$, and $8 \times 10^{-3} \mathrm{M} \mathrm{CaCl}_{2}$ caused a mean decrease of $55.4( \pm 14.4) \%$. These results show that the concentration of calcium in the suffusate bathing the tibialis anterior muscle during fluorescent intravital microscopy significantly influences the vasoactive response of arterioles to light. Further investigation of the mechanisms of light-induced effects on microcirculation during fluorescent intravital microscopy will become important as this technique is used more widely in the study of the microcirculation of solid tissues and organs. 1992 Academic Press, Inc.
\end{abstract}

\section{INTRODUCTION}

Fluorescent intravital microscopy using epi-illumination from a halogen light source represents a significant technological advance in the study of the microcirculation of tissues in vivo. Fluorescent intravital microscopy permits the study of solid organs or tissues that are too thick or dense to transilluminate such as the liver or muscles of locomotion such as the tibialis anterior. We and others have shown (Messina and Carlson, 1991; Damon and Duling, 1984; Reed and Miller, 1987; Dawson et al., 1987; Sonina and Khayutin, 1989; Rosenblum et al., 


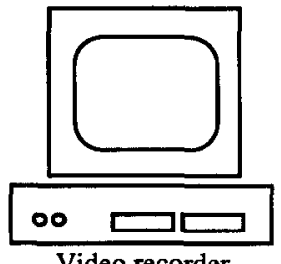

Video recorder
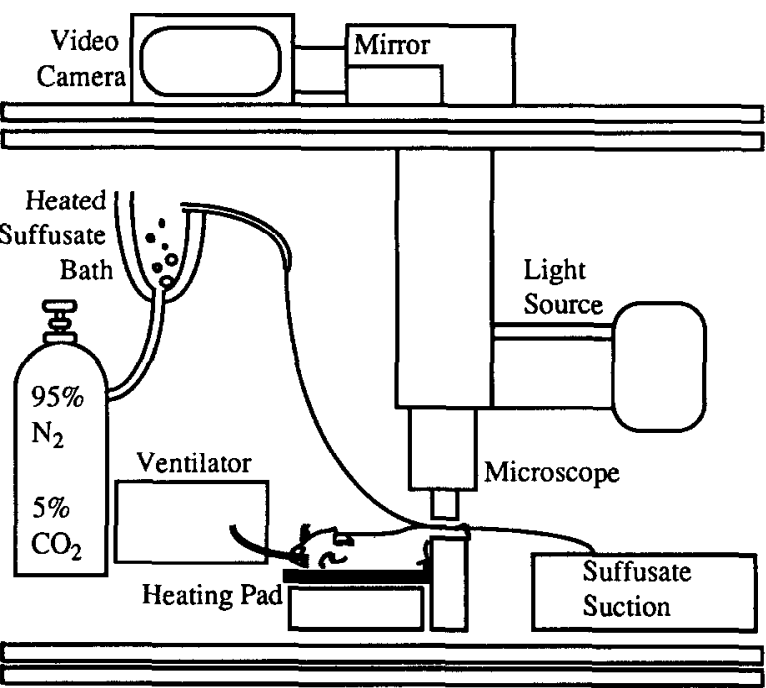

Fig. 1. Schematic illustration of intravital microscope.

1987) that arterioles may undergo a transient vasoactive response when exposed to light during fluorescent intravital microscopy. The effect of light on arteriolar reactivity described in different preparations is variable, usually consisting of vasodilation, vasoconstriction, or the initiation of vasomotion. This variability is due in part to the specific wavelength studied and the specific experimental preparation.

There is a rapidly expanding body of knowledge concerning the effect of light on vascular smooth muscle reactivity. Furchgott and associates $(1961,1985)$ have shown that isolated arterial smooth muscle placed in active contraction in a water bath relaxes upon exposure to light. This relaxation is reversible and is a hyperbolic function of the radiation intensity. Light-induced vasoreactivity in these studies is potentiated by low temperatures and is least effective at wavelengths greater than $450 \mathrm{~nm}$. These and other studies (Karlsson and Axelsson, 1984) have shown that an increase in cyclic guanosine monophosphate accompanies photorelaxation. Light can also cause vascular smooth muscle constriction particularly in the presence of photo-sensitizing drugs, such as hematoporphyrins and free fluorescein (Vanhoutte, 1980; Rosenblum et al., 1987; Reed et al., 1987; Sonina and Khayutin, 1989).

Damon and Duling (1984) noted that the vasoactive response of arterioles in the cremaster muscle upon exposure to light during fluorescent intravital microscopy occurred both in the presence and in the absence of a fluorochrome and over a variety of excitation wavelengths. Using fluorescent intravital microscopy to study the tibialis anterior muscle of the hamster, we have found similar effects of light on arteriolar reactivity. The most common effect is the initiation of a form of vasomotion characterized by rhythmic, diffuse, intermittent contractions of the arteriolar wall which occasionally progress in a wave-like fashion along the arteriole. We have also found this vasoactive response to fluorescent light to be dependent on the duration of light exposure and the wavelength of light used, 
similar to the findings of Damon and Duling (1984). Finally, we have noted little or no effect of light on arteriolar reactivity during fluorescent intravital microscopy of regenerating arterioles (Messina and Carlson, 1991) and an exaggerated vasoactive response to light by arterioles in ischemic muscle (Messina, 1990).

Because the predominant vasoactive effect of light on arterioles in the hamster tibialis anterior muscle is to initiate vasomotion and because vasomotion has been shown to be a calcium-dependent process (Colantuoni et al., 1984; DeClerck et al., 1989), we hypothesized that the type and frequency of the vasoactive response by arterioles to light during fluorescent intravital microscopy are calcium dependent. In order to test this hypothesis, we quantitated the type and the frequency of vasoactive responses by arterioles to light during fluorescent intravital microscopy of the tibialis anterior muscle in the presence and absence of calcium within the suffusate bathing the muscle. In addition, we determined whether the presence or absence of calcium also influenced the reactivity of these arterioles to the vasoactive agents adenosine and norepinephrine. Finally, in order to characterize more fully the effect of the concentration of calcium in the suffusate on arteriolar reactivity, we determined the effect of increases in the concentration of calcium in the suffusate on the resting diameter of the arterioles.

\section{MATERIALS AND METHODS}

Preparation. Syrian golden hamsters (Charles River, Wilmington, MA) weighing between 90 and $100 \mathrm{~g}$ were anesthetized with sodium pentobarbital $(0.1 \mathrm{mg} / \mathrm{g}$ body wt). The right carotid artery and the left jugular vein were cannulated, and a polyethylene endotracheal tube was inserted into the trachea. The hamsters were transferred to the stage of a Wayland-Frasher intravital microscope (Wayland, 1971). To maintain continuous anesthesia, a constant infusion (0.0078-0.0130 $\mathrm{ml} / \mathrm{min}$ ) of sodium pentobarbital $(9 \mathrm{mg} / \mathrm{ml})$ was given through the cannula in the jugular vein. The polyethylene endotracheal tube was connected to a mechanical ventilator set at a rate and volume sufficient to maintain arterial blood gases within normal physiologic limits. Arterial blood gases were obtained for $\mathrm{pH}$ and gas analysis from the cannula in the carotid artery. A rectal temperature probe was placed and connected to a servo-controlled heating pad, which maintained rectal temperature at $37^{\circ}$. The right or left lower leg was fixed to a Plexiglass platform in a horizontal plane (Fig. 1). The skin and tendinous portion of the biceps femoris muscle were incised directly over the tibiaiis anterior muscle. At all times after exposure of the muscle, the muscle was bathed in a suffusate that had a millimolar composition of $\mathrm{NaCl}, 130.9 ; \mathrm{KCl}, 4.7 ; \mathrm{CaCl}_{2}, 2.0 ; \mathrm{MgSO}_{4}, 1.2 ; \mathrm{NaHCO}_{3}, 20.0$. The flow rate of the suffusate $(5 \mathrm{ml} / \mathrm{min})$ was adjusted to provide a temperature of $35^{\circ}$ over the muscle. The temperature was measured frequently throughout the procedure with a beaded thermistor probe inserted directly into the suffusate over the muscle. The suffusate was equilibrated with gas containing $5 \% \mathrm{CO}_{2}$ and $95 \%$ $\mathrm{N}_{2}$ to maintain the $\mathrm{pH}$ between 7.38 and 7.42. The entire preparation was enclosed in a plastic tent to ensure that the oxygen concentration surrounding the preparation was maintained below $10 \mathrm{~mm} \mathrm{Hg}$ to minimize any influence of oxygen on the reactivity of the arterioles. Arterial blood pressure was monitored continuously throughout the procedure to adjust the anesthetic to a level that ensured a constant blood pressure throughout the experiment. 
After an equilibration period of $50 \mathrm{~min}$, bovine serum albumin (Sigma Chemical Co., St. Louis, MO) conjugated to rhodamine B $(0.2 \mathrm{ml})$ (excitation $567 \mathrm{~nm}$; emission $584 \mathrm{~nm}$ ) (Molecular Probes, Eugene, OR) was given intravenously. This fluorochrome was prepared as recommended by the manufacturer (Molecular Probes). Lissamine rhodamine, dissolved in a drop of dimethylformamide, was added to a solution of bovine serum albumin $20 \mathrm{mg} / \mathrm{ml}$ in carbonate-buffered saline ( $\mathrm{pH} 8.8$ to 9.4). The mixture was stirred in the dark for $1 \mathrm{hr}$ at room temperature. Subsequently, the solution was concentrated using Centiprep 30 concentrators (Amicon, Danvers, MA). This concentrate was washed and resuspended repeatedly using the carbonate-buffered saline. The final concentrate was suspended in saline at a concentration of $40 \mathrm{mg} / \mathrm{ml}$ and passed through multiple filters to sterilize the solution.

The preparation was illuminated with a 100-W mercury lamp (Wild Leitz, Inc., Cincinnati, $\mathrm{OH}$ ) connected to a incident light illuminator (American Optical, Buffalo, NY) (Fig. 1). The light passed first through an excitation filter $(546 \mathrm{~nm})$ and second through a dichroic mirror $(560 \mathrm{~nm})$ and then reflected back through a barrier filter that transmitted light above $515 \mathrm{~nm}$ to a relay lens and subsequently to the television camera. Two heat filters (Model KGB12 Leitz) were used; these filters also functioned as neutral density filters (5\% each) and removed all wavelengths of light $<450 \mathrm{~nm}$. A $20 \mathrm{X}$ immersion objective (NA $=0.33$, Nikon Inc., Garden City, NY) was used. A closed-circuit video system which consisted of a Dage-SIT Model 66 video camera (Dage-MTI, Inc., Michigan City, IN), a Panasonic 4-Head VHS videocassette recorder, and a Panasonic 12 -in. high-resolution video monitor was used to view the microcirculation. Final magnification was 1100X.

Experimental Protocol. In order to determine the effect of the presence $(2 \times$ $10^{-3} M$ ) or absence of calcium in the suffusate on the type and frequency of the vasoactive response of arterioles to light during fluorescent intravital microscopy as well as on the response of these arterioles to adenosine and norepinephrine, the following protocol was used. In each hamster the entire experimental protocol was executed in either the presence $\left(2 \times 10^{-3} M\right)(n=7)$ or the absence of calcium $(n=10)$ in the suffusate. In each hamster we first examined the responsiveness of arterioles $(10-20 \mu \mathrm{m})$ to the topical application of adenosine $\left(10^{-4}, 10^{-5}, 10^{-6} \mathrm{M}\right)$ and norepinephrine $\left(10^{-7}, 5 \times 10^{-8}, 10^{-8} \mathrm{M}\right)$ by measuring the diameter of arterioles (Messina and Carlson, 1991). The appropriate concentrations of the vasoactive agents were achieved by adding adenosine and norepinephrine to the suffusate reservoir. The arterioles were recorded on videotape $2 \mathrm{~min}$ after exposure of the vasoactive agent. The reservoir was flushed thoroughly between different concentrations of the vasoactive substances. The arteriole was then allowed to return to its original diameter between applications. Changes in arteriolar diameter were observed on a high-resolution video monitor and recorded on a videotape. During playback of the videotape, the diameter of the arterioles was measured with calipers when the border of the column of fluorescent plasma was at its sharpest and at its widest diameter. The percentage change in diameter was determined from the ratio of the change in diameter to the initial diameter. The dimensions measured were calibrated by measuring the video image of a stage micrometer (Graticules, Ltd., Tonbridge, K, UK) during video playback.

In the same hamster we then determined the type and frequency of light-induced 
vasoreactivity of the arterioles. Every $15 \mathrm{~min}$ for $1 \mathrm{hr}$ four randomly chosen fields containing two or three arterioles were each exposed to the fluorescent light for $30 \mathrm{sec}$. Four different fields were studied at each observation point in order to avoid any cumulative light-induced injury. The type and frequency of vasoactivity during fluorescent microscopy of the arterioles were characterized in each field as no effect, vasomotion, partial vasoconstriction, or total vasoconstriction (vessel occlusion). In most fields there were two or three arterioles which reacted uniformly to the fluorescent light exposure. Because the tibialis anterior muscle is a thick, solid muscle it cannot be transilluminated. Therefore, between exposures to the fluorescent light, no observations could be made concerning the vasoactive state of the arterioles.

In a separate group of hamsters $(n=7)$ we determined the effect of the suffusate calcium concentration on resting arteriolar diameter. In these experiments the calcium concentration in the suffusate was increased stepwise from 0 to $2 \times 10^{-3}$ to $4 \times 10^{-3}$ to $8 \times 10^{-3} \mathrm{M}$. After flushing the bath, a suffusate containing the appropriate concentration of calcium was added to the suffusate reservoir and changes in arteriolar diameter were measured at 5 and 30 min after the change in calcium concentration. The doses of calcium were always added from the lowest to the highest concentration because the highest dose of calcium changed the responsiveness of the arterioles. The change in arteriolar diameter was determined as described above.

Statistical analysis. Results are reported as the mean \pm SEM of the percentage change in diameter of the arterioles. The mean change in arteriolar diameter (nonnormalized) for each concentration of adenosine and norepinephrine when the suffusate contained $2 \times 10^{-3} \mathrm{M} \mathrm{CaCl}_{2}$ was compared by an unpaired $t$ test to the mean change in arteriolar diameter at the same concentration when there was no calcium in the suffusate. The effect of the calcium concentration of the suffusate on the incidence of vasoactive responses by arterioles to light was tested statistically by Likelihood Ratio $\chi^{2}$. Using the approach of Zeger and Liang (1986, 1987) for analysis of longitudinal data, the effect of the calcium concentration on arteriolar reactivity was also analyzed using a regression analysis because there might be less variance among observations made on the same hamster than among observations performed on different days on different hamsters. The method employed controlled for the extra binomial variation. The result is an estimate of the regression coefficient that treats the correlation of observations on the same hamster as a nuisance variable. We used robust estimates of variance because it adjusted for the uncertainty in the structure of the correlation matrix. This statistical analysis was performed under a SAS macro (GEE-1). A $P<0.05$ was considered significant.

\section{RESULTS}

The concentration of calcium in the suffusate significantly influenced the frequency of vasoactive response by arterioles to light during fluorescent intravital microscopy of the tibialis anterior muscle (Table 1). The frequency of vasoreactivity of arterioles was $77 \%$ in the presence of calcium $\left(2 \times 10^{-3} \mathrm{M}\right)$ and was significantly less, $58 \%$, when calcium was absent from the suffusate. The type of vasoactive response to light was similar for the two experimental conditions (Table 
TABLE 1

Effect of Suffusate Calcium Concentration on the Frequency and Type of Vasoactive Response of Arterioles of Tibialis Anterior during Fluorescent Intravital Microscopy

\begin{tabular}{lcc}
\hline & \multicolumn{2}{c}{ Suffusate calcium concentration } \\
\cline { 2 - 3 } & $\begin{array}{c}0 \mathrm{~m} M \\
\text { of fields observed } \\
(n)\end{array}$ & $\begin{array}{c}2 \times 10^{-3} M \\
\text { of fields observed } \\
(n)\end{array}$ \\
\hline Frequency of Vasoactivity & & \\
Vasoactivity present & $58 \%^{*}$ & $77 \%$ \\
& $(113)$ & $(105)$ \\
Vasoactivity absent & $42 \%^{*}$ & $23 \%$ \\
& $(83)$ & $(31)$ \\
Type of Vasoactivity & & $71 \%$ \\
Vasomotion & $81 \%$ & $24 \%$ \\
Partial vasoconstriction & $18 \%$ & $6 \%$ \\
Total vasoconstriction & $2 \%$ & \\
\hline
\end{tabular}

${ }^{*} P<0.05$ vs $2 \times 10^{-3} \mathrm{mM}$ calcium (nonnormalized data); $n$, number of fields; mean resting diameter of arterioles $11.4 \mu \mathrm{m}\left(0 \mathrm{~m} M \mathrm{CaCl}_{2}\right)$ and $10.4 \mu \mathrm{m}\left(2 \times 10^{-3} \mathrm{MCaCl}_{2}\right)$.

1). The most frequent type of vasoactive response observed was vasomotion which accounted for $71 \%$ of the episodes of vasoactivity in the group of hamsters in which there was calcium in the suffusate and for $81 \%$ of the episodes of vasoactivity that occurred in the group of hamsters in which there was no calcium in the suffusate. Partial arteriolar vasoconstriction occurred in $24 \%$ of the episodes of vasoreactivity when calcium was in the suffusate and in $18 \%$ of the episodes of vasoactivity when there was no calcium in the suffusate. Thus, the presence of 2 $\times 10^{3} \mathrm{M} \mathrm{CaCl}_{2}$ in the suffusate significantly increased the frequency but not the type of vasoreactivity observed during light exposure.

Although the presence of $2 \times 10^{-3} \mathrm{M} \mathrm{CaCl}_{2}$ significantly influenced the frequency of vasoactivity during fluorescent light exposure, removing calcium from the suffusate did not change the responsiveness of these arterioles to the vasoactive agents adenosine and norepinephrine (Table 2). No statistically significant differences in the response of arterioles to the three different concentrations of adenosine and norepinephrine were observed. At the highest doses of each vasoactive agent, the percentage change in the diameter of the arterioles was nearly identical. The mean percentage increase in diameter upon exposure to adenosine in the group of hamsters in which there was $2 \times 10^{-3} \mathrm{M} \mathrm{CaCl}_{2}$ in the suffusate was $74.6( \pm 5.3) \%$ and was $76.9( \pm 5.0) \%$ in the group in which there was no $\mathrm{CaCl}_{2}$ in the suffusate. Similarly, in response to the highest dose of norepinephrine $\left(10^{-7} M\right)$, the mean decrease in diameter in the group in which there was $\mathrm{CaCl}_{2}$ in the suffusate was $27.2( \pm 2.4) \%$ and was $27.5( \pm 3.2) \%$ in the group in which there was no calcium in the suffusate.

In order to characterize more fully the effect of the suffusate calcium concentration on arteriolar reactivity, we measured the percentage change in the diameter of arterioles of the tibialis anterior $5 \mathrm{~min}$ after exposure to a range of calcium concentrations in the suffusate bathing the muscle (Table 3). A concentration of $2 \times 10^{-3} \mathrm{M} \mathrm{CaCl}_{2}$ caused a significant decrease in the mean diameter of the 
TABLE 2

Effect of Suffusate Calcium Concentration on Responsiveness of Arterioles of the Tibialis ANTERIOR to Adenosine and Norepinephrine

\begin{tabular}{|c|c|c|}
\hline \multirow[b]{2}{*}{ Vasoactive agent } & \multicolumn{2}{|c|}{ Suffusate calcium concentration } \\
\hline & $0 \mathrm{~m} M$ & $\begin{array}{c}2 \times 10^{-3} \\
\mathrm{~m} M\end{array}$ \\
\hline \multicolumn{3}{|l|}{ Adenosine } \\
\hline \multicolumn{3}{|l|}{$10^{-6} M$} \\
\hline $\begin{array}{l}\text { Increase (\%) } \\
(n)\end{array}$ & $\begin{array}{c}17.8( \pm 3.6) \\
(10)\end{array}$ & $\begin{array}{c}13.0( \pm 6) \\
(7)\end{array}$ \\
\hline \multicolumn{3}{|l|}{$10^{-5} M$} \\
\hline $\begin{array}{l}\text { Increase }(\%) \\
(n)\end{array}$ & $\begin{array}{c}38.7( \pm 4.4) \\
(10)\end{array}$ & $\begin{array}{c}24.3( \pm 7.0) \\
(6)\end{array}$ \\
\hline \multicolumn{3}{|l|}{$10^{-4} M$} \\
\hline $\begin{array}{l}\text { Increase }(\%) \\
(n)\end{array}$ & $\begin{array}{c}76.9( \pm 5.1) \\
(10)\end{array}$ & $\begin{array}{c}74.6( \pm 5) \\
(7)\end{array}$ \\
\hline \multicolumn{3}{|l|}{$\begin{array}{l}\text { Norepinephrine } \\
\qquad 10^{-8} M\end{array}$} \\
\hline $\begin{array}{l}\text { Decrease (\%) } \\
(n)\end{array}$ & $\begin{array}{c}10.1( \pm 2.4) \\
(9)\end{array}$ & $\begin{array}{c}8.9( \pm 2.7) \\
(7)\end{array}$ \\
\hline \multicolumn{3}{|l|}{$5 \times 10^{-8} M$} \\
\hline $\begin{array}{l}\text { Decrease }(\%) \\
(n)\end{array}$ & $\begin{array}{c}23.0( \pm 3) \\
(10)\end{array}$ & $\begin{array}{c}17.5( \pm 5.9) \\
(6)\end{array}$ \\
\hline \multicolumn{3}{|l|}{$10^{-7} M$} \\
\hline $\begin{array}{l}\text { Decrease }(\%) \\
(n)\end{array}$ & $\begin{array}{c}27.5( \pm 3.2) \\
(10)\end{array}$ & $\begin{array}{c}27.2( \pm 2.4) \\
(6)\end{array}$ \\
\hline
\end{tabular}

Note. Values are expressed as mean \pm SEM for percentage change in diameter. Statistics performed on nonnormalized data. ( $n$, number of hamsters). Mean resting diameter of control arterioles was $11.4 \mu \mathrm{m}$.

TABLE 3

Effect of Changes in Calcium Concentration on Resting Arteriolar Diameters

Calcium concentration Percentage decrease*

(n)

\begin{tabular}{cc}
\hline $2 \times 10^{-3} M$ & $11.6( \pm 3) \%^{* *}$ \\
$4 \times 10^{-3} M$ & $41.2( \pm 12) \%^{* *}$ \\
& $(7)$ \\
$8 \times 10^{-3} M$ & $55.4( \pm 14) \%^{* *}$ \\
& $(7)$
\end{tabular}

Note. $n$, numbers of artcrioles, mean diameter $11.7 \mu \mathrm{m}$.

* Percentage decrease in diameter mean resting diameter.

${ }^{* *} P<0.05$ vs $0 \mathrm{~m} M$ calcium Student's $t$ test, nonnormalized data. 
arterioles of $11.6( \pm 3.0) \%$ from that measured when there was no calcium in the suffusate. Further sequential increases in calcium suffusate concentrations above normal concentrations were associated with progressively larger decreases in arteriolar diameter. A concentration of $4 \times 10^{-3} M$ caused a mean decrease in the arteriolar diameter of $41.2( \pm 12.0) \%$ (range $0-100 \%, P<0.006)$. Finally, at a suffusate concentration of $8 \times 10^{-3} \mathrm{M}$, a mean decrease in the diameter of the arteriole of $55.4( \pm 14.4) \%(P<0.005)$ was observed.

In the same hamsters, in addition to the diameter changes measured at $5 \mathrm{~min}$ after exposure to the different $\mathrm{CaCl}_{2}$ concentrations, we also measured the diameter changes at $30 \mathrm{~min}$ after exposure. We assumed that at calcium concentrations other than that in the intravascular space, calcium might leach into or out of the suffusate and into the intravascular space and that this effect would be time dependent. The only significant difference between the responses to the $\mathrm{CaCl}_{2}$ concentrations at $5 \mathrm{~min}$ after exposure and $30 \mathrm{~min}$ after exposure occurred in the group in which there was $2 \times 10^{-3} \mathrm{M} \mathrm{CaCl}_{2}$ in the suffusate, in which a mean decrease in the diameter of $7.3( \pm 2.8) \%(P=0.03)$ occurred. At the other concentrations of the suffusate, there was no significant difference between the 5 and 30-min diameter measurements.

\section{DISCUSSION}

These results show that the concentration of calcium in the suffusate bathing the tibialis anterior muscle of the hamster during fluorescent intravital microscopy significantly influences the frequency of the vasoactive response of arterioles to light exposure (Table 1). The predominant vasoactive response of arterioles upon exposure to light during fluorescent intravital microscopy was the initiation of vasomotion, a diffuse rhythmic constriction and dilation of the arteriolar wall. The effect of the calcium in the suffusate was to increase the frequency of this vasoactive response to the exposure of light. Under the conditions of these experiments, we did not see extravasation of the fluorochrome outside the microcirculation as has been noted by others (Reed $e t$ al., 1988) as well as by us in the tibialis anterior muscle of the rat.

Although the presence of calcium in the suffusate increased the frequency of light-induced vasoreactivity, the presence or absence of calcium in the suffusate did not significantly influence the responsiveness of the arterioles to the vasoactive agents adenosine and norepinephrine. These results do not exclude the possibility that calcium concentrations other than those studied in this experiment might have significantly influenced the vasoactive response to these or other vasoactive agents. In addition, under the conditions of these experiments, other vasoactive agents may have been influenced by the concentration of calcium in the suffusate bathing the muscle. Finally, we did not exclude the possibility that calcium leached out of the intravascular space in the suffusate during conditions of "zero" calcium in the suffusate.

In addition to influencing the frequency of light-induced arteriolar vasoactivity, the suffusate calcium concentration affected resting arteriolar diameters (Table 3 ). The difference in resting diameter between zero added $\mathrm{CaCl}_{2}$ and $2 \times 10^{-3}$ $M \mathrm{CaCl}_{2}$ in the latter experiments was only $11 \%$. Such a small difference may in part explain why no difference was found in the responsiveness of arterioles to 
adenosine and norepinephrine (Table 2). Interestingly, in the hamsters in which these vasoactivity experiments were conducted a $10 \%$ difference in the mean control arteriolar diameters $(11.4 \mu \mathrm{mvs} 10.4 \mu \mathrm{m})$ for the zero added calcium group versus the $2 \times 10^{-3} \mathrm{M} \mathrm{CaCl}_{2}$ group was found. The significant effect of the calcium concentration of the suffusate shown in this in vivo study is similar to that observed in an in vitro study of larger $(60$ to $90 \mu \mathrm{m})$ arterioles by Hynes and Duling (1991).

The mechanism of light-induced vasoactive effects on large arteries and arterioles within the microcirculation is clearly complex and multifactoral, the effect being dependent upon the experimental conditions and the vessel being studied (Furchgott et al., 1955, 1961, 1984, 1985; Duling et al., 1984; Reed et al., 1987, 1988). Although some investigators have utilized light and free fluorescein or methylene blue to induce endothelial injury (Rosenblum et al., 1987), most studies show that the effect of light upon vascular reactivity is a direct smooth muscle effect independent of the endothelium (Furchgott $e t$ al., 1985). Others have shown that the effect of light on vascular reactivity can be different depending upon the wavelength of light studied, the intensity of the light, and the duration of the exposure. We did not examine these variables in these experiments; rather, the focus of these experiments was to characterize the effect of light during the specific conditions that are found during fluorescent intravital microscopy.

A variety of mechanisms have been put forth to explain the direct effect of light on vascular smooth muscle reactivity. Furchgott and associates (1955), who were one of the first to report the effect of light on vascular reactivity, have found that light, specifically ultraviolet radiation, acts on a photosensitive material within smooth muscle which produces a labile photo-induced relaxing factor which stimulates guanine monophosphate synthesis. We do not believe that this is a likely explanation for the mechanism of the light-induced vasoactive changes observed in this study for the following reasons. The experimental conditions employed by Furchgott and his colleagues are different than the conditions of our experiments. They studied isolated arterial smooth muscle strips from rabbit aorta. The response of the smooth muscle to light was one of photorelaxation rather than the initiation of vasomotion or vasoconstriction, and the maximal relaxatory effects were obtained at a wavelength of $310 \mathrm{~nm}$, which was a wavelength excluded in this study. In addition, photorelaxation has been shown under these conditions to be independent of changes in $\mathrm{pH}$ or in calcium concentrations (Furchgott et al., 1971). Under the experimental conditions of our experiments, all ultraviolet radiation was excluded, and the vasoactive effect of light was clearly calcium dependent. Thus, we believe that the mechanism of light-induced changes observed in this study is different from that observed by Furchgott and colleagues. This difference in the vasoactive effects of light strongly supports the concept that light may influence vessel reactivity by a variety of mechanisms depending on the type of smooth muscle studied and the experimental conditions.

We believe the effect of light on vasoreactivity of arterioles during intravital microscopy may be a direct effect on vascular smooth muscle which does not require the presence of a fluorochrome. Both Damon and Duling (1984) as well as Reed and Miller (1987) have shown the effect of light during fluorescent intravital microscopy to be independent of the presence of a fluorochrome. Since the arterioles of the tibialis anterior can be observed only with the use of a 
fluorochrome, we cannot make a similar judgment concerning the dependency of the light-induced changes on the presence of a fluorochrome. However, the dependency of light-induced effects on calcium concentrations shown in this study supports the hypothesis that the light-induced effect is a direct smooth muscle effect. The smooth muscle of the microvessels of skeletal muscle has resting tone and shows vasomotion in the presence of normal levels of extracellular calcium. These arterioles also have a particularly rapid transmembrane calcium flux which is tightly controlled by an intracellular regulatory mechanism. Recently, Hynes and Duling (1991) have shown that the calcium concentration in the bathing solution surrounding an arteriole significantly influenced cystolic and stored calcium concentrations within the smooth muscle, that the effect of change in calcium concentrations was rapid ( $T=1 / 2$ maximum of $14 \mathrm{~S})$, and that this effect of the calcium concentration of the bathing solution was much greater than changes in luminal calcium concentration.

Calcium can also affect vascular smooth muscle reactivity through other mechanisms such as oxygen free radical production. Calcium has been shown to potentiate the effect of the reduction in oxygen and, thus, free radical formation. In addition, the interaction of light and fluorescent compounds causes a photochemical reaction which can result in the generation of singlet oxygen (Foote, 1984). We do not believe that the generation of oxygen free radicals explains the vasoactive effects of light in our experiments because the predominant effect of free radical liberation on the microcirculation is to cause persistent vasodilatation, not the initiation of vasomotion or vasoconstriction. In addition, other vasoactive effects have not been shown to be blocked by hemoglobin or methylene blue or other free radical scavengers (Furchgott $e$ t al., 1984). We, in unpublished observations, have been unable to block the vasoactive effect of light with a variety of free radical scavengers. Others have shown that the predominant effect of free radicals generated under such conditions (Herrmann, 1982; Rosenblum et al., 1987) has been mediated by platelet aggregation and thrombus formation rather than a change in arteriolar reactivity. Because the predominant effect of light under the experimental conditions of this study was to induce vasomotion, and because vasomotion has been shown to be a calcium-dependent process, we believe the mechanism of light-induced arteriolar reactivity to be a direct smooth muscle effect which is dependent on extracellular calcium concentrations (Kuriyama $\boldsymbol{e t}$ al., 1971; DeClerck et al., 1989).

Fluorescent intravital microscopy represents an important technical advance in the study of the microcirculation of thick, solid tissues and organs. These results show that the light used in these studies may induce vasoactive effects that need to be taken into account when reporting the results of such studies. Clearly, when the duration and intensity of light during fluorescent intravital microscopy are not controlled, serious injury to the microcirculation can occur (Reed et al., 1987; Rosenblum et al., 1987; Damon and Duling et al., 1984). However, others have shown (Miller et al., 1982; Damon and Duling, 1984; Boric et al., 1987; Lund et $a l$. , 1984) that when the intensity of light and the duration of exposure are controlled, the effect on the microcirculation can be minimized. Nonetheless, further investigation of the mechanisms of light-induced effects on microcirculation during intravital microscopy will become important as this technique is utilized more widely in the study of the microcirculation of solid tissues and organs. 


\section{ACKNOWLEDGMENT}

We thank Mary Proctor for her help in the statistical analysis of the results and Cathy Blankenburg for her expert preparation of the manuscript.

\section{REFERENCES}

Boric, M. P., Roblero, J. S., and Duran, W. N. (1987). Quantitation of bradykinin-induced microvascular leakage of FITC-dextran in rate cremaster muscle. Microvasc. Res. 33, 397-412.

Colantuoni, A., Bertuglis, R., And Intaglietta, M. (1984). The effects of $\alpha$ - or $\beta$-adrenergic receptor agonists and antagonists and calcium entry blockers on the spontaneous vasomotion. Microvasc. Res. 28, 143-158.

Damon, D. H., And Duling, B. R. (1984). Distribution of capillary blood flow in the microcirculation of the hamster: An in vivo study using epifluorescent microscopy. Microvasc. Res. 27, 81-95.

Dawson, J. M., Trler, K. R., ANd Hudlicka, O. (1987). A comparison of the microcirculation in rat fast glycolytic and slow oxidative muscles at rest and during contractions. Microvasc. Res. 33, $167-182$.

De Clerck, F., Loots, W., Voeten, J., and JAnssen, P. A. J. (1989). Differential effects of verapamil and flunarizine on epinephrine-induced vasoconstriction and on spontaneous vasomotion of arterioles in skeletal muscle in the rat in vivo. J. Cardiovasc. Pharmacol. 13, 76-83.

FooTE, C. (1984). Mechanisms of photooxygenation. In "Porphyrin Localization and Treatment of Tumors" (D. R. Foiron and C. J. Gones, Eds.), pp. 3-18. A. R. Liss, New York.

Furchgott, R. F., Sleator, W., JR., McCaman, M. W., AND ElchlepP, J. (1955). Relaxation of arterial strips by light, and the influence of drugs on this photodynamic effect. J. Pharmacol. Exp. Ther. 113, 22-23.

FurChGott, R. F. (1971). Effects of various agents on photorelaxation of rabbil aortic strips. In "Physiology and Pharmacology of Vascular Neuroeffector Systems" (J. A. Bevan et al., Eds.), pp. 247-262. Karger, Basel.

Furchgott, R. F., Ehrreich, S. J., and Greendlatt, E. (1961). The photoactivated relaxation of smooth muscle of rabbit aorta. J. Gen. Physiol. 44, 499-519.

Furchgott, R. F., Martin, W., Cherry, P. D., Jothianandan, D., and Villani, G. M. (1985). Endothelium-dependent relaxation, photorelaxation and cyclic GMP. In "Vascular Neuroeffector Mechanisms" (J. A. Bevan, T. Godfraind, R. A. Maxwell, and P. M. Vanhoutte, Eds.), pp. 105114. Elsevier, New York.

Furchgott, R. F., Martin, W., Jothianandan, D., and Villani, G. M. (1984). Comparison of endothelium-dependent relaxation by acetylcholine and endothelium-independent relaxation by light in the rabbit aorta. In "Proceedings of IUPHAR 9th International Congress of Pharmacology, London, 1984" (W. Paton, J. Mitchell, and P. Turner, Eds.), Vol. 1, pp. 149-157. Macmillan, London.

Herrmann, K. S. (1983). Platelet aggregation induced in the hamster cheek pouch by a photochemical process with excited fluorescein isothiocyanate-dextran. Microvasc. Res. 26, 238-251.

Hynes, M. R., AND Duling, B. R. (1991). $\mathrm{Ca}^{2+}$ sensitivity of isolated arterioles from the hamster cheek pouch. Am. J. Physiol. 260, H355-H361.

Karlsson, J. O. G., Axelsson, K. L., and Andersson, R. G. G. (1984). Effects of ultraviolet radiation on the tension and the cyclic GMP level of bovine mesenteric arteries. Life Sci. 34, 15551563.

Kuniyama, H., Ohshima, K., and Sakamoto, Y. (1971). The membrane properties of the smooth muscle of the guinea pig portal vein in isotonic and hypertonic solutions. J. Physiol. 217, 179-199.

Lund, N., Damon, D. H., Damon, D. N., And Duling, B. R. (1987). Capillary grouping in hamster tibialis anterior muscles: Flow patterns, and physiological significance. Int. J. Microcirc. Clin. Exp. 5, 359-372.

Messina, L. M., and Carlson, B. M. (1991). Rapid and complete recovery of responsiveness to adenosine and norepinephrine by regenerating arterioles of the tibialis anterior muscle of the hamster after in situ autografting. Circ. Res. 68, 1660-1609.

Messina, L. M. (1990). In vivo assessment of acute microvascular injury after reperfusion of ischemic tibialis anterior muscle of the hamster. J. Surg. Res. 48, 615-621. 
Miller, F. N., Joshua, I. G., ANd Andreson, G. L. (1982). Quantitation of vasodilator induced macromolecular leakage by in vivo fluorescent microscopy. Microvasc. Res. 24, 56-67.

ReEd, M. W. R., Miller, F. N., AND Weiman, J. J. (1987). Microcirculatory effects of photoactivation of dihematoporphyrin ether. In "Microcirculation- $-\Lambda$ U Update" (M. Tsuchiya et al., Eds.), Vol. 2, pp. 825-826. Elsevier, B.V.

Reed, M. W. R., AND MilleR, F. N. (1988). Importance of light dose in fluorescent microscopy. Microvasc. Res. 36, 104-107.

Rosenblum, W. I., Nelson, G. H., and Povlishock, J. T. (1987). Laser-induced endothelial damage inhibits endothelium-dependent relaxation in the cerebral microcirculation of the mouse. Circ. Res. 60, 169-176.

Sonina, R. S., And Khayutin, V. M. (1989). The dilation of arterioles in frog submaxillaris muscle caused by visible light. Int. J. Microcirc. Clin. Exp. 8, 143-158.

VAnhoutte, P. M. (1980). Physical factors of regulation. In "Handbook of Physiology" (D. F. Bohr, A. P. Somlyo, and H. V. Sparks, Jr., Eds.), pp. 464-465. Williams \& Wilkins, Baltimore.

Wayland, H., AND Frasher, W. (1971). Intravital microscopy on the basis of telescopic principles: Design and application of an intravital microscopy for microvascular and neurophysiological studies. Int. Symp. Mod. Physiol. Sci. (Munich), 124-145.

ZEGER, S. L., AND LIANG, K. (1986). Longitudinal data analysis for discrete and continuous outcomes. Biometrics 42, 121-130.

Zeger, S. L., Liang, K., AND Albert, P. S. (1988). Models for longitudinal data: A generalized estimating equation approach. Biometrics 44, 1049-1060. 